\title{
Pathogenicity and In Planta Mycotoxin Accumulation Among Members of the Fusarium graminearum Species Complex on Wheat and Rice
}

\author{
Rubella S. Goswami and H. Corby Kistler
}

First and second authors: Department of Plant Pathology, University of Minnesota, St. Paul 55108; and second author: USDA-ARS Cereal

Disease Laboratory, 1551 Lindig Street, University of Minnesota, St. Paul 55108.

Accepted for publication 23 July 2005.

\begin{abstract}
Goswami, R. S., and Kistler, H. C. 2005. Pathogenicity and in planta mycotoxin accumulation among members of the Fusarium graminearum species complex on wheat and rice. Phytopathology 95:1397-1404.

Fusarium head blight (FHB), or scab, is a destructive disease of small grains caused by members of the Fusarium graminearum species complex, comprised of at least nine distinct, cryptic species. Members of this complex are known to produce mycotoxins including the trichothecenes deoxynivalenol (DON) along with its acetylated derivatives and nivalenol (NIV). In this study, 31 strains, belonging to eight species of this complex and originating from diverse hosts or substrates, were tested for differences in aggressiveness and mycotoxin production. Large variation among strains, both in terms of their aggressiveness and the ability to
\end{abstract}

ABSTRACT produce trichothecenes on a susceptible cultivar of wheat was found; variation appears to be a strain-specific rather than species-specific characteristic. While pathogenicity was not influenced by the type of mycotoxin produced, a significant correlation was observed between the amount of the dominant trichothecene (DON and its acetylated forms or NIV) produced by each strain and its level of aggressiveness on wheat. Some isolates also were tested for their ability to infect rice cv. M201, commonly grown in the United States. While tested strains were capable of infecting rice under greenhouse conditions and causing significant amount of disease, no trichothecenes could be detected from the infected rice florets.

Additional keywords: F. acaciae-mearnsii, F. asiaticum, F. austroamericanum, F. boothii, F. meridonale, F. mesoamericanum, Gibberella zeae.
Fusarium head blight (FHB), also known as scab, is an extremely destructive disease that has reached epidemic proportions in North America and many other parts of the world in the past decade (reviewed in 18). This disease affects wheat, barley, and other small grains both in temperate and semitropical areas. The major causal organisms of this disease belong to the recently described Fusarium graminearum species complex (32), whereby the genealogical concordance phylogenetic species recognition (GCPSR) approach (36) was used to delineate species limits among strains morphologically characterized as Fusarium graminearum Schwabe (teleomorph Gibberella zeae (Schweinitz) Petch). This led to the identification of seven phylogenetically distinct entities designated initially as lineages 1 to 7 (31). Some of these lineages appeared to be localized on particular continents or geographic regions (31). More recent work, including a larger collection of strains and DNA sequence comparisons from a total of 11 genes at six independent genetic loci, supported the existence of nine distinct, cryptic species within the $F$. graminearum species complex $(32,39)$. These species now have been formally described and named (32) with $F$. graminearum sensu stricto retained for the species most commonly associated with FHB worldwide

Corresponding author: H. C. Kistler; E-mail address: hckist@umn.edu

The use of trade, firm, or corporation names in this publication is to accurately describe experiments for the information of the reader. Such use does not constitute an official endorsement or approval by the United States Department of Agriculture or the Agricultural Research Service of any product or service to the exclusion of others that may be suitable.

\section{DOI: $10.1094 /$ PHYTO-95-1397}

This article is in the public domain and not copyrightable. It may be freely reprinted with customary crediting of the source. The American Phytopathological Society, 2005. (lineage 7). F. asiaticum (formerly known as $F$. graminearum lineage 6) is the most common cause of FHB in certain regions of China and other portions of Asia $(16,31,32)$. The other species, along with their previous lineage designation, include $F$. austroamericanum (lineage 1), F. meridionale (lineage 2), F. boothii (lineage 3), F. mesoamericanum (lineage 4), F. acaciae-mearnsii (lineage 5), F. cortaderiae (lineage 8), and F. brasilicum (previously not given a lineage designation).

The threat posed by these pathogens is multifaceted. Besides causing yield and quality losses due to sterility of the florets and formation of discolored, withered and light test-weight kernels, the pathogens also produce significant levels of deleterious mycotoxins including trichothecenes and the estrogenic mycotoxin zearalenone $(24,26,27,34)$. Trichothecene toxins such as deoxynivalenol (DON), also known as vomitoxin, and nivalenol (NIV) are sesquiterpenoids that are potent inhibitors of eukaryotic protein biosynthesis and have been implicated in a number of human and animal mycotoxicoses $(34,37)$. Therefore, even low levels of these toxins in raw grain can make them deemed unfit for human or animal consumption. DON has also been identified as a virulence factor on wheat and maize $(4,12,19)$.

Individuals in the $F$. graminearum species complex produce predominantly either DON or its C-4 oxygenated derivative, nivalenol (NIV); DON producers also make acetyl ester derivatives of DON at the 3- and 15-position oxygens. Thus, three strain-specific profiles of trichothecene metabolites, called "chemotypes" (2), are observed for strains in the $F$. graminearum species complex and related species (39): NIV (NIV producers), 3ADON (DON producers that also make predominantly 3-acetyl, DON), and $15 \mathrm{ADON}$ (DON producers that also make predominantly 15acetyl, DON). Among the two main forms of trichothecenes produced by members of the species complex, NIV is almost 10 times more toxic to animals than DON (27). 
Genetic variation among isolates belonging to the $F$. graminearum species complex is an extensively described fact $(5,7,8$, $10,13,17,38)$. Some studies describe variation in culture characteristics (5) and mycotoxin production $(3,38)$. Recently, Xue et al. (40) reported variation in aggressiveness among 12 strains of F. graminearum in Canada. Host of origin is also reported to be a factor adding to the variability within these species (7). Reports on the correlation between aggressiveness and mycotoxin production by members of the $F$. graminearum species complex vary in their conclusions. For example, Adams and Hart (1) concluded that DON and 15ADON are not pathogenicity or virulence factors on maize. On the other hand, on wheat, trichothecene nonproducing mutants are reduced in virulence $(12,19)$ and in their ability to spread in the wheat head after causing initial infection (4). The influence of mycotoxin chemotype in determining pathogenicity of strains on a particular host has also been reported (8). However, most of these studies on differences in pathogenicity and aggressiveness did not distinguish strains potentially belonging to different species and have been limited to geographically localized collections of strains. Therefore, variation among these pathogens in their aggressiveness on wheat on a global scale and the role of mycotoxins in aggressiveness is still unclear.

The focus of studies on scab in North America largely has been directed towards the disease on wheat, barley, and maize. However, rice (Oryza sativa) is another important cereal crop where these pathogens cause significant losses in other parts of the world. In fact, scab was one of the first diseases of rice to be recorded (21). It has been known to exist in most of the rice growing regions in the world (21) and in recent years there have been several reports of scab on rice in Asian countries $(7,11,22)$. $\mathrm{Scab}$ is usually not considered a serious threat to rice but can be

TABLE 1. Strains included in this study (32)

\begin{tabular}{|c|c|c|c|}
\hline $\begin{array}{l}\text { Taxon (Fusarium } \\
\text { graminearum clade } \\
\text { lineage designation) }\end{array}$ & $\begin{array}{l}\text { NRRL } \\
\text { strain } \\
\text { number }\end{array}$ & Host/substrate & Geographic origin \\
\hline F. acaciae-mearnsii & 26752 & Acacia mearnsii & South Africa \\
\hline F. acaciae-mearnsii & $26754 \mathrm{~T}^{\mathrm{a}}$ & Acacia mearnsii & South Africa \\
\hline F. acaciae-mearnsii & 26755 & Acacia mearnsii & South Africa \\
\hline F. asiaticum & 6101 & Barley & Japan \\
\hline F. asiaticum & $13818 \mathrm{~T}$ & Barley & Japan \\
\hline F. asiaticum & 26156 & Wheat & Shanghai Prov.-China \\
\hline F. asiaticum & 28720 & Corn & Nepal \\
\hline F. asiaticum & 28303 & Wheat & Japan \\
\hline F. asiaticum & 28306 & Wheat & Japan \\
\hline F. austroamericanum & $2903 \mathrm{~T}$ & $\ldots$ & Brazil \\
\hline F. austroamericanum & 28585 & Herbaceous vine & Venezuela \\
\hline F. austroamericanum & 28718 & Corn & Brazil \\
\hline F. boothii & 29020 & Corn & South Africa \\
\hline F. boothii & $26916 \mathrm{~T}$ & Corn & South Africa \\
\hline F. boothii & 29011 & $\ldots$ & South Africa \\
\hline F. boothii & 29105 & Corn & Nepal \\
\hline F. cortaderiae & $29297 \mathrm{~T}$ & Pampas grass & New Zealand \\
\hline F. cortaderiae & 29306 & Corn & New Zealand \\
\hline F. graminearum & 5883 & Corn & Ohio-USA \\
\hline F. graminearum & 6394 & Millet & Hungary \\
\hline F. graminearum & 13383 & Corn & Iran \\
\hline F. graminearum & 28063 & Corn & Michigan-USA \\
\hline F. graminearum & 28336 & Wheat & Ohio-USA \\
\hline F. graminearum & 28439 & $\begin{array}{l}\text { Florida } \\
\text { leatherleaf fern }\end{array}$ & Netherlands \\
\hline F. graminearum & 29169 & Wheat & Kansas-USA \\
\hline F. graminearum & 31084 & Corn & Michigan-USA \\
\hline F. meridonale & $28436 \mathrm{~T}$ & Orange twig & New Caledonia \\
\hline F. meridonale & 28723 & Corn & Nepal \\
\hline F. meridonale & 29010 & Soil & South Africa \\
\hline F. meridonale & $28721^{\mathrm{b}}$ & Corn & Nepal \\
\hline F. mesoamericanum & 29148 & Cissus rhombifolia & Pennsylvania-USA \\
\hline
\end{tabular}

a $\mathrm{T}=$ ex-holotype strain.

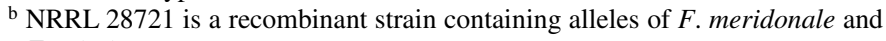
F. asiaticum. severe under conditions favorable to disease (33). To our knowledge, there are no reports of scab on rice in the United States. However, it has been observed in an unrelated species, Zizania palustris (wild rice), grown under conditions of high humidity similar to that of cultivated rice (29).

The objective of this study was to assess variation in aggressiveness and trichothecene production of strains belonging to eight species of the $F$. graminearum complex. A collection of 31 strains isolated from diverse hosts and substrates from all over the world was used. Some members of the F. graminearum species complex commonly known to affect wheat were also evaluated for their ability to infect a rice cultivar grown in the United States and to produce mycotoxin.

\section{MATERIALS AND METHODS}

Strains. Considering the broad host range attributed to the $F$. graminearum species complex $(9,15,20,31)$, we included strains from a number of different host species in our studies. The 31 single-spored strains selected for the study along with their geographic origin and host or substrates are listed in Table 1. Methods for storing and culturing these strains have been previously described $(30,32)$.

Plant growth conditions. Six seeds of wheat cv. Norm were planted in 6-in. pots filled with steamed topsoil. They were grown in a greenhouse at approximately $20 \pm 3^{\circ} \mathrm{C}$ with $16 \mathrm{~h}$ supplemental light until anthesis. Plants were watered daily and treated with Nutricote 13-13-13 micronutrients, Type 100 (Plantco Inc., Brampton, ON) 3 weeks after planting.

Seeds for rice cv. M201 were provided by G. Milus, University of Arkansas. Rice was planted and fertilized in the same manner as used for wheat except that the pots were placed in planters with 7 to $12 \mathrm{~cm}$ of water throughout the growing period. The plants were kept at $30 \pm 3^{\circ} \mathrm{C}$ in a growth cabinet with a mixture of fluorescent and incandescent bulbs with a 14-h day length until flowering.

Pathogenicity tests. Stored cultures were grown on mung bean agar (14) for 10 days at room temperature under a combination of fluorescent and near-UV light bulbs with a 12-h photoperiod. After this period, macroconidia were collected by flooding the petri dish with sterile water and washing them off the surface of the media. Thereafter, they were counted under a microscope with a hemacytometer. Inoculum was prepared containing $1 \times$ $10^{6}$ macroconidia in $1 \mathrm{ml}$ of sterile water, to which Triton 60 was added to a final concentration of $0.01 \%$ for better adherence of inoculum to the host tissue.

Wheat heads of susceptible cv. Norm were inoculated at earlyto mid-anthesis with approximately $1 \times 10^{4}$ conidia. Using a micropipette, $10 \mu \mathrm{l}$ of the inoculum was applied to the lower or central spikelet, from which the awns had been previously removed. After inoculation, the plants were placed in a humidity chamber for $72 \mathrm{~h}$ and then transferred to a greenhouse maintained at approximately $27^{\circ} \mathrm{C}$. The highly aggressive strains NRRL 29169 and NRRL 31084 (also known as PH-1) were used as positive controls for basal and central spikelet inoculation, respectively. The mean number of symptomatic spikelets from heads inoculated with NRRL 29169 and 31084 did not significantly differ $(P=0.96)$ when compared using a $t$ test (two-tailed distribution with two-sample unequal variance). Wheat heads inoculated with sterile water (containing $0.01 \%$ Triton 60 ) were used as negative (mock-inoculated) controls. Inoculation treatments consisted of a total of 10 plants with five plants within a pot and two pots per treatment. Every experiment was repeated twice each with the basal and central spikelet inoculation techniques for at least a total of four replicates. Head blight was rated 14 days after inoculation (dai) by counting the number of spikelets showing disease symptoms (necrosis and/or bleaching of palea/lemma) as described by Proctor et al. (35). A total of 10 spikelets on each 
wheat head were evaluated for the presence of disease symptoms. In experiments where basal inoculation was performed, scoring began with the basal inoculated spikelet moving upwards to the 10 th spikelet. Where the central spikelet was inoculated, five upper and four lower spikelets were scored. The inoculated spikelet was collected, weighed, and stored for mycotoxin analysis. This method was used for all 31 strains. Five strains, namely NRRL 31084, 29169, 26156, 28303, and 28306, were selected for further analysis to assess the spread of the fungus and mycotoxin accumulation within the wheat heads. In these cases, one glume from each scored spikelet of the inoculated wheat head was surface sterilized by soaking in 10\% household bleach for $2 \mathrm{~min}$ followed by four rinses in sterile water with subsequent plating on mung bean agar. The growth of the fungus was recorded after $48 \mathrm{~h}$. The remaining portion of the spikelet was weighed and used for mycotoxin analysis.

To determine relative aggressiveness of fungal strains to wheat, the mean number of symptomatic spikelets from each replicate was normalized with respect to the positive control. A two-tailed $t$ test with two-sample unequal variance was used to test whether strains were significantly different $(P \leq 0.01)$ from the positive control. The correlation coefficient $(R)$ and coefficient of determination $\left(R^{2}\right)$ were used to determine the relationship between strain aggressiveness and mean trichothecene concentration determined for wheat infected with each strain. The significance of $R$ was then confirmed by calculating the $t$ value.

To measure aggressiveness on rice cv. M201, panicles at earlyto mid-anthesis were sprayed to run-off with a hand-held sprayer containing a conidial suspension $\left(1 \times 10^{4}\right.$ spores per $\mathrm{ml}$ in $0.01 \%$ Triton 60). Inoculations were performed on 10 panicles with each strain, and this procedure was replicated once. As for wheat, the plants were kept in a dew chamber for $72 \mathrm{~h}$ after inoculation and then transferred to the greenhouse for evaluation of disease symptoms 14 days later. Plants inoculated with NRRL 31084 and sterile water were chosen as positive and negative controls, respectively. Disease assessment on rice was conducted by categorizing the florets in each panicle into three groups (Fig. 1A to C) and counting the number of florets in each group. Two samples from panicles inoculated with each strain containing six florets per sample were weighed and used for mycotoxin analysis. To verify that the symptoms observed were being caused by the inoculated strain, 20 symptomatic and 20 nonsymptomatic surface-sterilized florets were plated on mung bean agar following the procedure used for wheat. Growth of the fungus from the florets was recorded after $48 \mathrm{~h}$. In order to fulfill Koch's postulates, cultures growing from infected rice florets were isolated and single-spored cultures were established. These cultures were used for re-infection of both rice and wheat in a manner similar to that used in the earlier experiments.

Mycotoxin analysis. Wheat spikelets or rice florets were collected for analysis to determine the concentration of trichothecenes (DON, 3ADON, 15ADON, and NIV) present. Each sample consisting of a single wheat spikelet or six rice florets was weighed and placed in a 1-dram glass vial capped with a screw cap and extraction was carried out by soaking it in $1.5 \mathrm{ml}$ of acetonitrile/water (84:16, vol/vol) with shaking for $24 \mathrm{~h}$. Subsequently, approximately $1.3 \mathrm{ml}$ of the extract was passed through a minicolumn (1-ml tuberculin syringe packed with $150 \mathrm{mg}$ of 1:3 [wt/wt] C18/aluminum oxide) and $1 \mathrm{ml}$ of the elutant was transferred to a 0.5 -dram vial. The samples were evaporated to dryness using nitrogen. Twenty-five microliters of TMS reagent $(N$ trimethylsilylimidazole [TMSI]/trimethylchlorosilane [TMCS], 100:1) was added to the vial. After $10 \mathrm{~min}, 200 \mu \mathrm{l}$ of isooctane was added followed by $200 \mu \mathrm{l}$ of water to quench the reaction. The samples were vortexed so that the milky isooctane layer became transparent. This upper isooctane layer was finally transferred into a 1.5 -ml gas chromatography vial for gas chromatography/mass spectrometry (GC/MS) analysis.
GC/MS analysis was carried out using a Shimadzu GCMSQP2010 system with an AOC-20s autosampler and AOC-2oi autoinjector (Shimadzu Corporation, Kyoto, Japan). Selected ion monitoring (SIM) mode was used for mycotoxin analysis. SIM was applied to detect the characteristic ions of DON with fragment ion ( $\mathrm{m} / \mathrm{z}$ value) of 235.10 as the target ion and 259.10 and 422.10 as the reference ions; 3ADON with fragment ion of 377.10 as the target ion and 392.10 as the reference ion; 15ADON with fragment of 193.00 as the target ion and 392.10 as the reference ion; and NIV with fragment ion of 289.05 as the target ion and 379.10 as the reference ion. The GC column used was a J\&W DB-5ms type with $0.25-\mu \mathrm{m}$ film thickness, $250-\mu \mathrm{m}$ internal diameter, and $30 \mathrm{~m}$ in length (Agilent Technologies, Palo Alto, $\mathrm{CA}$ ). The temperature program used for the GC/MS analysis had an initial column temperature of $150^{\circ} \mathrm{C}$ for $1 \mathrm{~min}$ and was increased to $280^{\circ} \mathrm{C}$ at a rate of $30^{\circ} \mathrm{C} / \mathrm{min}$ and held for $5 \mathrm{~min}$. The injection temperature was kept at $300^{\circ} \mathrm{C}$, and column flow rate was $1 \mathrm{ml} / \mathrm{min}$.

\section{RESULTS}

Pathogenicity on wheat. The pathogenic potentials of strains belonging to eight species of the $F$. graminearum species com-
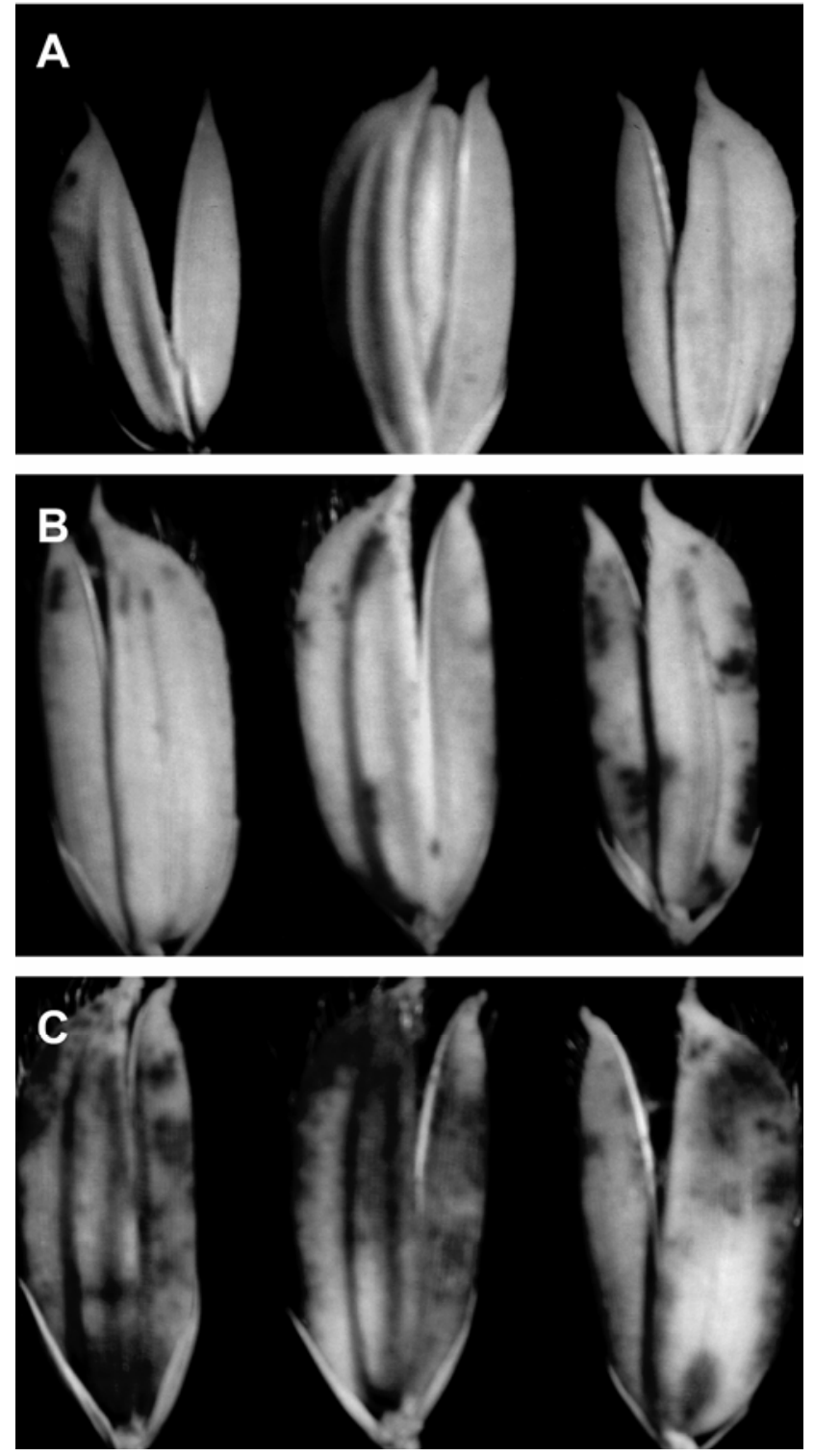

Fig. 1. Categories used for assessment scab on rice: A, Nonsymptomatic florets; B, florets showing $<50 \%$ lesion area; and $\mathbf{C}$, florets showing $>50 \%$ lesion area. 
plex, including five ex-holotype strain specimens, were ascertained by the ability of disease to spread within the wheat head from a single point of inoculation. Interestingly, our tests found that all eight species contained strains that could spread and cause considerable disease in spikelets above and below the point of inoculation, including strains isolated from nongramineous hosts (Fig. 2A; Table 1). All strains of the $F$. graminearum species complex were capable of producing detectable quantities of trichothecenes (Fig. 2B), though the amounts produced by individual strains varied greatly.

Comparisons between the average numbers of symptomatic spikelets in wheat heads inoculated at the basal spikelet compared with inoculation of a central spikelet revealed no significant difference at 14 dai $(P>0.05)$ for all strains. Data from these experiments were therefore pooled for use in determining the capacity of strains to spread on the wheat head. Based on their similarity to the positive control (NRRL 31084), these strains were divided into two groups. The group that possessed aggressiveness similar to NRRL $31084(P \geq 0.05)$ was called highly aggressive, whereas the others $(P \leq 0.05)$ were categorized as less aggressive. Each species included in the study, with the exception of $F$. meridionale (represented in the study by three strains) and F. mesoamericanum (represented in the study by only one strain), contained at least one strain that could be assigned to the highly

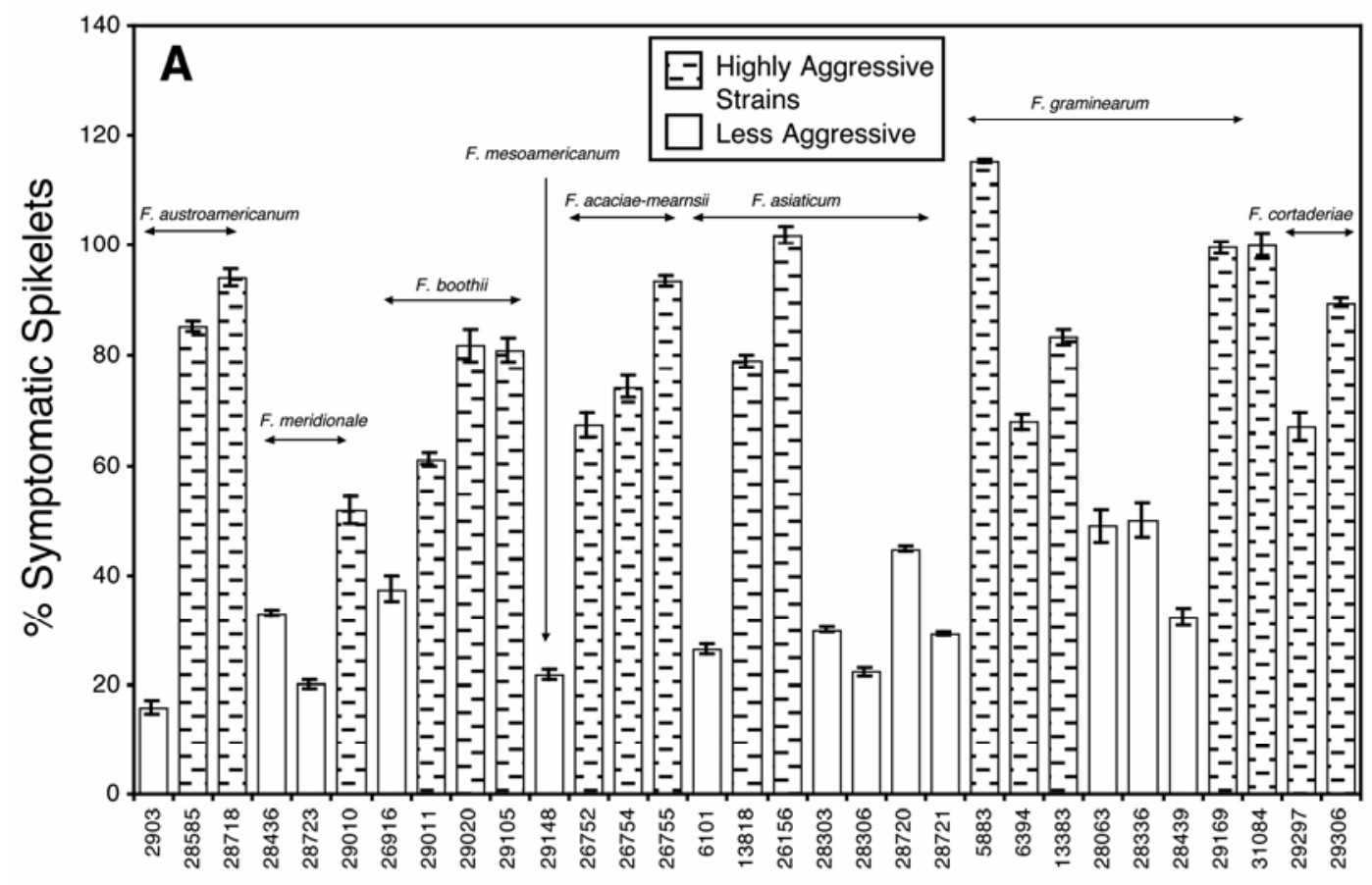

Strains

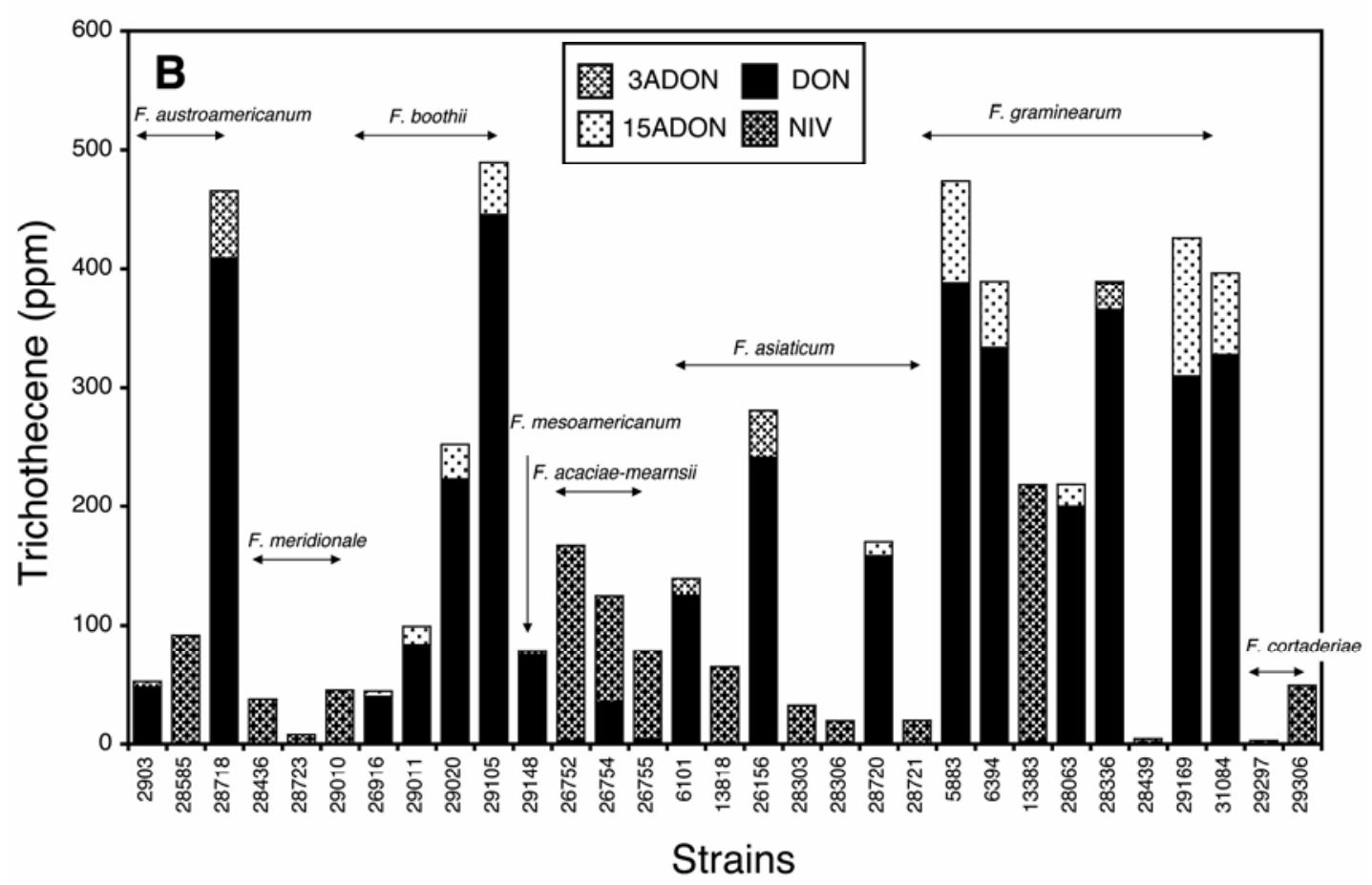

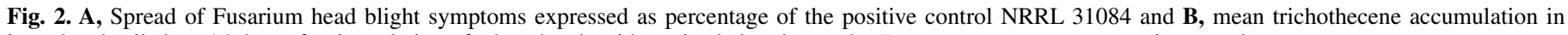
inoculated spikelets, 14 days after inoculation of wheat heads with strains belonging to the Fusarium graminearum species complex. 
aggressive category. However, species with highly aggressive strains also contained less aggressive strains and no particular pattern could be observed in their distribution. Within wheat heads, symptoms were observed to spread in both directions with a slightly higher spread observed towards the lower spikelets. This trend was more pronounced in less aggressive strains.

Analysis of trichothecene accumulation on wheat heads infected with the strains included in this study showed that the strains belonging to $F$. meridionale, $F$. acaciae-mearnsii, and $F$. cortaderiae produced NIV. Members of $F$. boothii were DON producers. The other species, F. austroamericanum, F. asiaticum, and $F$. graminearum, contained strains producing both DON and NIV chemotypes. Strains belonging to $F$. graminearum, which had been isolated from cereal crops in the United States, were found to be primarily the $15 \mathrm{ADON}$ chemotype except one strain, NRRL 28336, which was 3ADON. Geographically, one of the two NIV producing members of $F$. graminearum originated from Iran and the other had been isolated in the Netherlands. Among the DON producing strains, members of $F$. boothii were the 15ADON chemotype, whereas members of $F$. austroamericanum were 3ADON. $F$. asiaticum contained both 3ADON and $15 \mathrm{ADON}$ producing strains. Despite differences in the chemotype among these species, a significant correlation between the trichothecene accumulation and the ability to spread on the wheat head was observed (Fig. 3). This correlation was stronger for DON producing strains than those producing NIV. Since the 3ADON and 15ADON producers also accumulated a significant amount of DON, the correlation between DON and the number of diseased spikelets was also estimated for these strains. Additionally, the NIV producers occasionally produced low levels of DON. On comparing the quantity of mycotoxin produced by the different chemotypes, the range of NIV and DON produced by the strains was 3 to $216 \mathrm{ppm}$ and 39 to $461 \mathrm{ppm}$, respectively. Thus, the concentration range of NIV was lower than the range of DON. However, NIV producers were as aggressive as DON producers. Phenotypically, a variation in symptoms developing on wheat heads infected with strains that produced lower levels of mycotoxin was noted. Wheat heads inoculated with less aggressive strains appeared to show more necrosis in the form of blackish lesions on the infected spikelets rather than bleaching and deformation of awns which was commonly seen in plants infected with the highly aggressive strains (data not shown).

To examine in more detail the progress of symptoms, growth of the fungus, and the accumulation of trichothecenes in inoculated wheat, five strains, NRRL 31084, 29169, 26156, 28303, and 28306 , belonging to $F$. graminearum and $F$. asiaticum were chosen. The occurrence of symptoms, presence of the fungus, and trichothecene levels were determined in five spikelets above and four below the point of inoculation on the wheat head (Fig. 4). For the less aggressive strains, NRRL 28303 and 28306, the fungus spread beyond symptomatic spikelets and occasionally could be shown to grow from nonsymptomatic spikelets farthest from the point of inoculation (i.e., spikelet number 1 and 10). Conversely, for the highly aggressive strains, NRRL 31084, 29169, and 26156 , spikelets farthest from the point of inoculation often were symptomatic, yet no fungus could be detected in the symptomatic tissue. For the less aggressive strains, the accumulation of trichothecenes in spikelets occurred in the inoculated spikelet and rarely in the spikelets above and below the point of inoculation, even if they were symptomatic. For highly aggressive strains, the accumulation of trichothecenes was largely correlated with the symptomatic spikelets.

Pathogenicity on rice. Pathogenicity tests on rice were conducted using the same five strains selected for detailed studies on wheat. Spraying of fungal spores on the rice panicle was found to be the most efficient and effective method for inoculation on rice. Symptoms observed on rice 14 dai consisted of black and brown lesions spreading over the surface of the florets with almost no bleaching. Due to differences in the expression of the disease on rice compared with wheat, a new methodology was designed for scoring and categorizing the diseased florets in order to compare strains. Florets were divided into three categories according to the approximate percentage of floret surface covered with necrotic lesions, (Fig. 1) namely, $<50,>50$, and $0 \%$ (considered nonsymptomatic). There were significant differences among the strains in
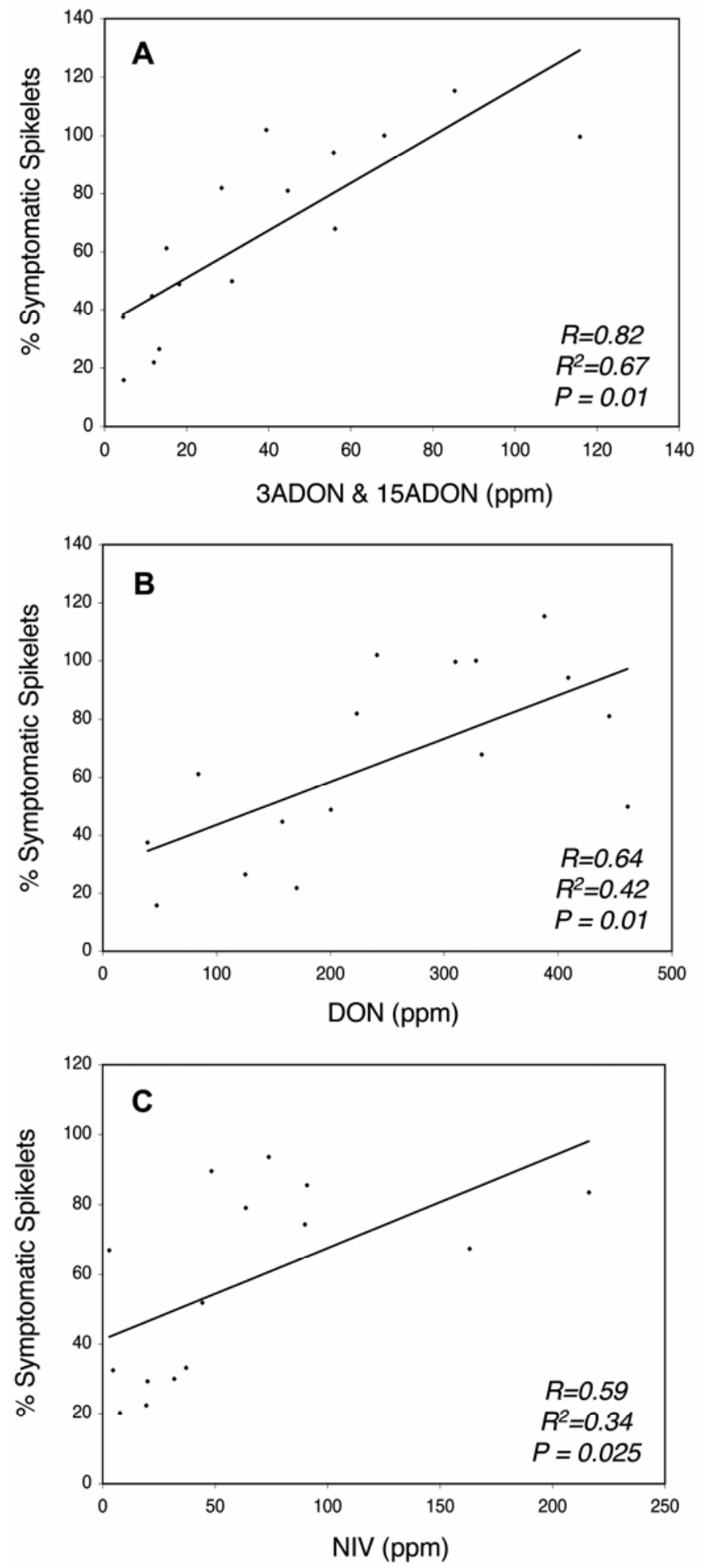

Fig. 3. Correlation between trichothecene accumulation and disease symptom expression. Concentration of A, 3ADON and 15ADON (for deoxynivalenol [DON] producers that also make predominantly 3-acetyl and 15-acetyl, DON), B, DON, and C, nivalenol (NIV) and symptomatic spikelets expressed as percentage of the positive control NRRL 31084, 14 days after inoculation. 
the percentage of florets having $>50 \%$ lesions and the trend was found to be similar to that observed in wheat (Fig. 5). Strains that had been classified as highly aggressive in wheat produced more diseased tissue in rice. Surprisingly, no trichothecenes were detected in infected rice with any of the strains tested in spite of the fact that all strains produced detectable amounts in wheat (Fig. 4). Previous reports also have shown that two of these strains produce trichothecenes when grown on autoclaved rice
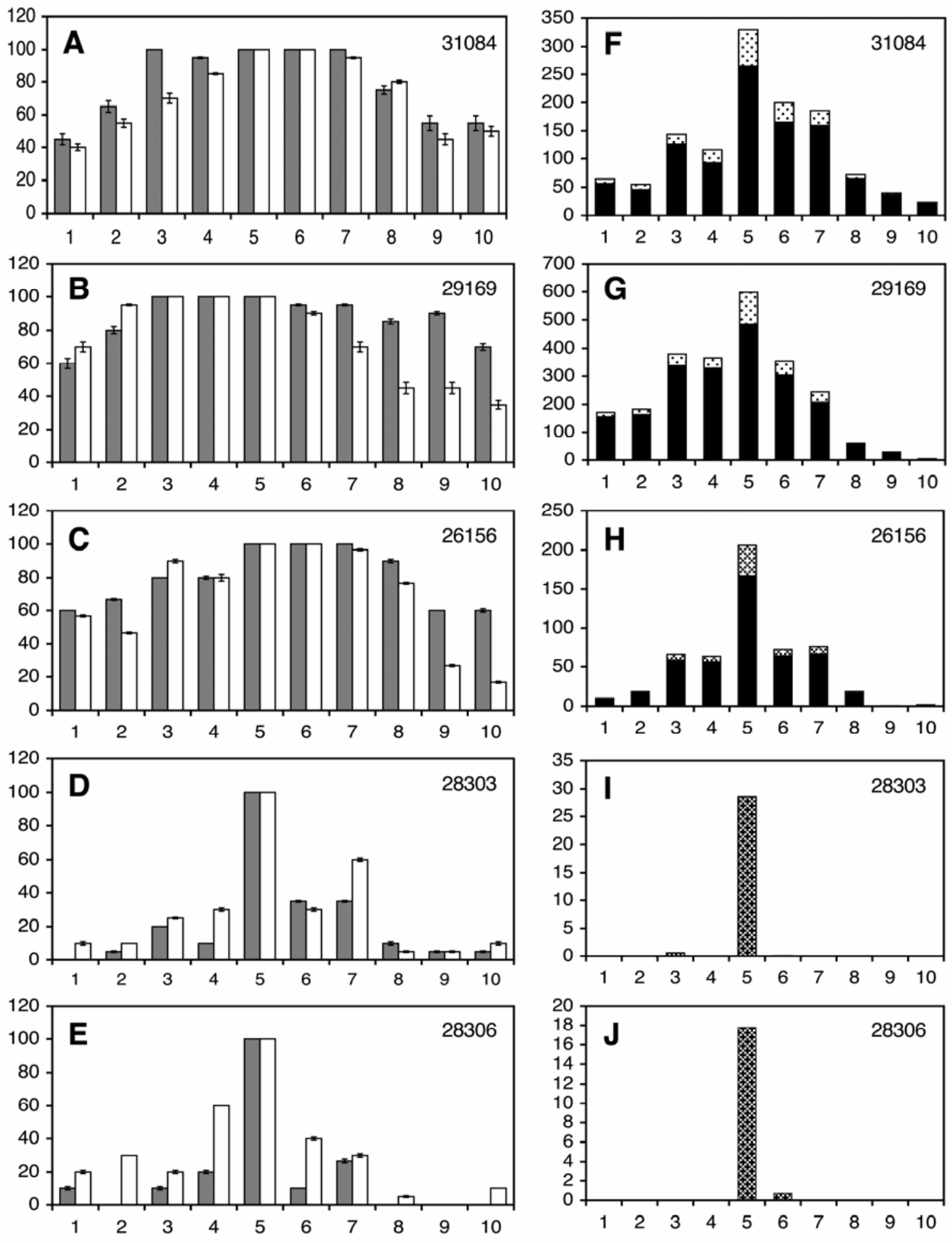

\section{Symptomatic Spikelets

Fig. 4. Disease spread and trichothecene production on wheat heads inoculated with strains belonging to the Fusarium graminearum species complex. A to E, Occurrence of Fusarium head blight symptoms and fungus; $\mathbf{F}$ to J, mean trichothecene concentration 14 days after inoculation of wheat heads with strains belonging to the $F$. graminearum species complex. $x$ axis denotes spikelet position (inoculation done at fifth spikelet), and $y$ axis denotes percentage of spikelets showing symptoms or fungal growth (A to $\mathbf{E}$ ) or trichothecene accumulation in ppm (F to $\mathbf{J})$. Note differences in scale for trichothecene concentrations among strains. 
culture (31). The strains retained the capability to cause FHB on wheat and produce trichothecenes when re-isolated from rice.

\section{DISCUSSION}

The results obtained from this study suggest that the capacity of strains within the $F$. graminearum complex to spread and cause disease on wheat is more likely to be a strain-specific rather than species-specific characteristic. The two species sampled most extensively, $F$. asiaticum and $F$. graminearum, each contained individuals of all three chemotypes: NIV, 3ADON, and 15ADON. We predict further sampling of the remaining species would likely identify individuals of each chemotype. The pathogenicity of the strains tested however did not appear to be influenced by the type of trichothecene produced. Rather, a significant correlation was observed between the level of the dominant trichothecenes (DON and its acetylated forms or NIV) produced by each strain and its level of aggressiveness on wheat. Previous reports comparing the aggressiveness of DON and NIV producing strains have been contradictory $(8,25)$. However, our study strongly suggests that it is the amount rather than the type of trichothecene produced that functions as a major determinant of aggressiveness on wheat. Our results are in agreement with previous studies showing that trichothecenes, which have been previously identified as virulence factors $(23,35)$, have a significant role in determining the aggressiveness of the pathogen. Highly aggressive strains have been found to produce higher levels of trichothecene irrespective of their chemotype. The fact that NIV has been shown to be more toxic than DON (27) could explain the observation that the maximum amount of trichothecene quantified from infected spikelets was higher for DON (461 ppm) than for NIV (216 ppm) producers with similar levels of aggressiveness.

Findings from experiments evaluating the spread of the fungus in relation to the symptoms suggested that for highly aggressive strains, occurrence of symptomatic spikelets showed a trend similar to the spread of the fungus although symptoms often were found to spread further from the point of inoculation on the wheat head than the fungus itself (Fig. 4A to C). Conversely, in less aggressive strains, the fungus could be isolated from nonsymptomatic spikelets away from the point of inoculation (Fig. 4D and E). This may be due to a difference in the primary mode of symptom development among strains, which also could relate to differences in aggressiveness. In less aggressive strains that also produce lesser amounts of mycotoxin, the spread of symptoms is more likely caused by the direct effect of the fungus colonizing host tissue rather than the effect of virulence factors like trichothecenes. Highly aggressive strains, on the other hand, have the added capacity of producing higher levels of trichothecenes that may be translocated in the plant and facilitate symptom development in advance of the growing fungus. This observation is supported by earlier findings demonstrating that trichothecene toxins could be found in tissues not colonized by the fungus (6).

The capacity of strains isolated from wheat and corn to cause disease on rice without the accumulation of detectable amounts of trichothecenes is surprising. In rice, symptoms of disease consist solely of necrotic lesions; blighting and bleaching symptoms closely associated with trichothecene production (28) are not seen. However, variation in symptom expression on rice was seen and characterized by the presence of necrotic lesions of varying sizes on the florets. This necessitated the development of a new method to effectively quantify disease expression on this host. Since spray inoculation was used on rice, differences among strains in the ability to cause symptoms in host florets may lie either in the differential ability to initially infect plants or to further develop and cause necrosis once infection has occurred. Previous literature on members of the $F$. graminearum species complex isolated from cultivated rice in Korea mentions that NIV producers could be the predominant chemotype affecting this host under agricultural conditions (22). This report was based on trichothecene analysis from field isolates grown on rice culture medium. However, studies by Walker et al. (38) on wheat have shown that in vitro and in vivo toxin production is not correlated. Desjardins et al. (11) also were unable to detect trichothecenes from rice seed samples in Nepal, but some of the isolated strains did produce mycotoxins when grown on rice cultures. They did not test these strains on other host plants and mentioned that the traditional methods of grain drying and storage could prevent the accumulation of trichothecenes in Nepalese rice. Our results indicate that scab caused on rice under greenhouse conditions may progress without the detectable accumulation of trichothecenes. The aggressive strains included in our study (NRRL 31084, 29169 , and 26156) on rice were proficient DON producers, and even the less aggressive strains (NRRL 28303 and 28306) could produce detectable amounts of NIV on wheat. Thus, our results suggest that strains that commonly infect wheat are capable of infecting a rice cultivar grown in the United States under favorable conditions even though they do not produce detectable amounts of trichothecenes. One possible explanation for this observation may be that rice possesses a detoxification mechanism capable of metabolizing trichothecenes to a form not detected by our GC/MS assay. Another possible explanation could be that the fungus is unable to produce trichothecenes because rice lacks a factor required for induction of trichothecene biosynthesis in the fungus or contains a factor that represses trichothecene biosynthesis. Apparently, the ability to prevent toxin accumulation is not present or is lost in autoclaved rice grains used in the rice culture medium, supporting the hypothesis of an active interaction between the host and the pathogen or the presence of a heat labile inhibitory compound.

Our studies show that there is significant variation among strains of the $F$. graminearum species complex in their ability to cause FHB on wheat. This variability in the pathogen in some instances could present difficulties to the diagnosis of the disease in the field and the timely application of control measures. The threat posed by this group of pathogens is compounded by the fact that even less aggressive strains produce trichothecenes in levels above the permissible limits of consumption by humans or animals. The existence of such large variability also emphasizes the need for breeders to include a wide range of strains in their screenings for selection of disease resistant varieties. The finding that some highly pathogenic members of the $F$. graminearum complex which cause scab on wheat are also able to infect rice, though they may not be capable of producing mycotoxins on rice, increases the risk of carry-over of this pathogen without detection. It also draws our attention to a potential new reservoir of the pathogen, which could serve as a source of inoculum. Moreover, discovering the mechanism by which Fusarium infection occurs in rice without the accumulation of detectable trichothecenes

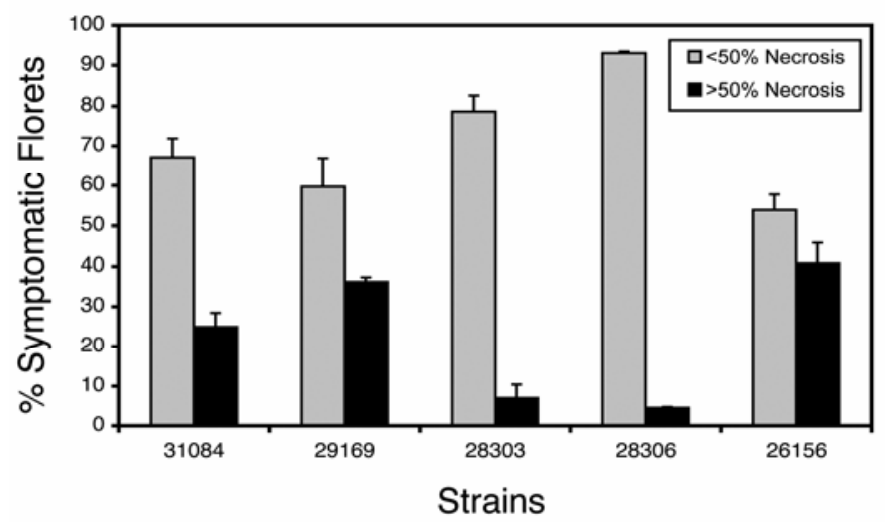

Fig. 5. Necrosis 14 days after infection in rice panicles inoculated with strains belonging to the Fusarium graminearum species complex. 
could be important in developing FHB resistance for other crops. Such information provides new insights into the global ecology and life history of this fungus and could prove critical to the development of comprehensive and effective disease and mycotoxin management.

\section{ACKNOWLEDGMENTS}

We thank W. Xie and Y. Dong (University of Minnesota) and B. Tacke (North Dakota State University) for mycotoxin analysis of the samples; G. Milus (University of Arkansas) for providing rice seed; K. Hilburn for help with growing plant material for research as well as K. Takamura and J.'N. O'Brian for assistance in sample preparation for mycotoxin analysis; L. Gale for presubmission review of the manuscript; K. O'Donnell and T. Ward for providing strains and helpful dialog; and J. Morrison for assistance with photography and layout. This work was supported by a grant from the U.S. Wheat and Barley Scab Initiative to H. C. Kistler.

\section{LITERATURE CITED}

1. Adams, G. C., and Hart, L. P. 1989. The role of deoxynivalenol and 15acetyldeoxynivalenol in pathogenesis by Gibberella zeae, as elucidated through protoplast fusions between toxigenic and nontoxigenic strains. Phytopathology 79:404-408.

2. Anderson, D. W., Black, R. M., Lee, C. G., Pottage, C., Rickard, R. L., Sandford, M. S., and Webber, T. 1989. Structure-activity studies of trichothecenes: Cytotoxicity of analogues and reaction products derived from T-2 toxin and neosolaniol. J. Med. Chem. 32:555-562.

3. Atanassov, Z., Nakamura, C., Mori, N., and Kaneda, C. 1993. Mycotoxin production and pathogenicity of Fusarium species and wheat resistance to Fusarium head blight. Can. J. Bot. 72:161-167.

4. Bai, G. H., Desjardins, A. E., and Plattner, R. D. 2001. Deoxynivalenol nonproducing Fusarium graminearum causes initial infection, but does not cause disease spread in wheat spikes. Mycopathologia 15:91-98.

5. Bai, G. H., and Shaner, G. 1996. Variation in Fusarium graminearum and cultivar resistance to wheat scab. Plant Dis. 8:975-979.

6. Bushnell, W. R., Hazen, B. E., and Pritsch, C. 2003. Histology and physiology of Fusarium head blight. Pages 44-83 in: Fusarium Head Blight of Wheat and Barley. K. J. Leonard and W. R. Bushnell, eds. The American Phytopathological Society, St. Paul, MN.

7. Carter, J. P., Rezanoor, H. N., Desjardins, A. E., and Nicholson, P. 2000. Variation in Fusarium graminearum isolates from Nepal associated with their host of origin. Plant Pathol. 49:452-460.

8. Carter, J. P., Rezanoor, H. N., Holden, D., Desjardins, A. E., Plattner, R. D., and Nicholson, P. 2002. Variation in pathogenicity associated with the genetic diversity of Fusarium graminearum. Eur. J. Plant Pathol. 108:573583.

9. Cook, R. J. 1981. Fusarium diseases in wheat and other small grains in North America. Pages 39-52 in: Fusarium: Diseases, Biology, and Taxonomy. P. E. Nelson, T. A. Toussoun, and R. J. Cook, eds. Penn. State Univ. Press, University Park, PA.

10. Cumagun, C. J. R., Bodwen, R. L., Jurgenson, J. E., and Leslie, J. F. 2004. Genetic mapping of pathogenicity and aggressiveness of Gibberella zeae (Fusarium graminearum) toward wheat. Phytopathology 94:520- 526.

11. Desjardins, A. E., Manandhar, H. K., Plattner, R. D., Manandhar, G. G., Poling, S. M., and Maragos, C. M. 2000. Fusarium species from Nepalese rice and production of mycotoxins and gibberellic acid by selected species. Appl. Environ. Microbiol. 66:1020-1025.

12. Desjardins, A. E., Proctor, R. H., Bai, G. H., McCormick, S. P., Shaner, G., Buechley, G., and Hohn, T. M. 1996. Reduced virulence of trichothecene-nonproducing mutants of Gibberella zeae in wheat field tests. Mol. Plant-Microbe Interact. 9:775-781.

13. Dusabenyagasani, M., Dostaler, D., and Hamelin, R. C. 1999. Genetic diversity among Fusarium graminearum strains from Ontario and Quebec. Can. J. Plant Pathol. 21:308-314.

14. Evans, C. K., Xie, W., Dill-Macky, R., and Mirocha, C. J. 2000. Biosynthesis of deoxynivalenol in spikelets of barley inoculated with macroconidia of Fusarium graminearum. Plant Dis. 84:654-660.

15. Farr, D. F., Bills, G. F., Chamuris, G. P., and Rossman, A. Y. 1989. Fungi on Plants and Plant Products in the United States. The American Phytopathological Society, St. Paul, MN.

16. Gale, L. R., Hernick, C. A., Takamura, K., Chen, L.-F., and Kistler, H. C. 2002. Population analysis of Fusarium graminearum from wheat fields in eastern China. Phytopathology 92:1315-1322.

17. Gale, L. R. 2003. Population biology of Fusarium species causing head blight of grain crops. Pages 120-143 in: Fusarium Head Blight of Wheat and Barley. K. J. Leonard and W. R. Bushnell, eds. The American Phytopathological Society, St. Paul, MN.

18. Goswami, R. S., and Kistler, H. C. 2004. Heading for a disaster: Fusarium graminearum on cereal crops. Mol. Plant Pathol. 5:515-525.

19. Harris, L. J., Desjardins, A. E., Plattner, R. D., Nicholson, P., Butler, G., Young, Y. C., Weston, G., Proctor, R. H., and Hohn, T. M. 1999. Possible role of trichothecene mycotoxins in virulence of Fusarium graminearum on maize. Plant Dis. 83:954-960.

20. Inch, S., and Gilbert, J. 2003. The incidence of Fusarium species recovered from inflorescences of wild grasses in southern Manitoba. Can. J. Plant Pathol. 25:379-383.

21. Lee, F. N. 1992. Scab. Page 30 in: Compendium of Rice Diseases. R. K. Webster and P. S. Gunnell, eds. The American Phytopathological Society, St. Paul, MN.

22. Lee, Y. W., Jeon, J. J., Kim, H., Jang, I. Y., Kim, H. S., Yun, S. H., and Kim, J. G. 2004. Lineage composition and trichothecene production of Gibberella zeae population in Korea. Pages 117-122 in: New Horizon of Mycotoxicology for Assuring Food Safety. (Proc. Int. Symposium of Mycotoxicology in Kagawa 2003.) T. Yoshizawa, ed. Japanese Association of Mycotoxicology.

23. McCormick, S. 2003. The role of DON in pathogenicity. Pages 165-183 in: Fusarium Head Blight of Wheat and Barley. K. J. Leonard and W. R. Bushnell, eds. The American Phytopathological Society, St. Paul, MN.

24. McMullen, M., Jones, R., and Gallenberg, D. 1997. Scab of wheat and barley: A re-emerging disease of devastating impact. Plant Dis. 81:13401348 .

25. Miedaner, T., Reinbrecht, C., and Schilling A. G. 2000. Association among aggressiveness, fungal colonization, and mycotoxin production of 26 isolates of Fusarium graminearum in winter rye head blight. J. Plant Dis. Prot. 107:124-134.

26. Miller, J. D., Greenhalgh, R., Wang, Y., and Lu, M. 1991. Trichothecene chemotypes of three Fusarium species. Mycologia 83:121-130.

27. Mirocha, C. J., Pawlosky, R. J., Tong-Xia, Z., and Lee, Y. W. 1985. Chemistry and biological activity of Fusarium roseum mycotoxins. Pages 291-305 in: Trichothecenes and Other Mycotoxins. Proc. Int. Mycotoxin Symposium Sydney, Australia, 1984. J. Lacey, ed. John Wiley \& Sons, New York.

28. Muriuki, J. G. 2001. Deoxynivalenol and nivalenol in pathogenesis of Fusarium head blight in wheat. Ph.D. thesis. University of Minnesota.

29. Nyvall, R. F., Percich, J. A., and Mirocha, C. J. 1999. Fusarium head blight of cultivated and natural wild rice (Zizania palustris) in Minnesota caused by Fusarium graminearum and associated Fusarium spp. Plant Dis. 83:159-164.

30. O'Donnell, K., Cigelnik, E., and Nirenberg, I. 1998. Molecular systematics and phylogeography of the Gibberella fujikuroi species complex. Mycologia 90:465-493.

31. O’Donnell, K., Kistler, H. C., Tacke, B. K., and Casper, H. H. 2000. Gene genealogies reveal global phylogeographic structure and reproductive isolation among lineages of Fusarium graminearum, the fungus causing wheat scab. Proc. Natl. Acad. Sci. USA 97:7905-7910.

32. O'Donnell, K., Ward, T. J., Geiser, D. M., Kistler, H. C., and Aoki, T. 2004. Genealogical concordance between the mating type locus and seven other nuclear genes supports formal recognition of nine phylogenically distinct species within the Fusarium graminearum clade. Fungal Genet. Biol. 41:600-623.

33. Ou, S. H. 1972. Scab in Rice Diseases. Commonwealth Mycological Institute, Kew, Surrey, England.

34. Peraica, M., Radic, B., Lucic, A., and Pavlovic, M. 1999. Toxic effects of mycotoxins in humans. Bull. World Health Org. 77:754-766.

35. Proctor, R. H., Hohn, T. M., and McCormick, S. P. 1995. Reduced virulence of Gibberella zeae caused by disruption of a trichothecene toxin biosynthetic gene. Mol. Plant-Microbe Interact. 8:593-601.

36. Taylor, J. W., Jacobson, D. J., Kroken, S., Kasuga, T., Geiser, D. M., Hibbett, D. S., and Fisher, M. C. 2000. Phylogenetic species recognition and species concepts in fungi. Fungal Genet. Biol. 31:21-32.

37. Ueno, Y., and Ishii, K. 1985. Chemical and biological properties of trichothecenes from Fusarium sporotrichoides. Pages 307-316 in: Trichothecenes and Other Mycotoxins. Proc. Int. Mycotoxin Symposium, Sydney, Australia. J. Lacey, ed. John Wiley \& Sons, New York.

38. Walker, S. L., Leath, S., Hagler, W. M., and Murphy, J. P. 2001. Variation among isolates of Fusarium graminearum associated with Fusarium head blight in North Carolina. Plant Dis. 85:404-410.

39. Ward, T. J., Bielawski, J. P., Kistler, H. C., Sullivan, E., and O'Donnell, K. 2002. Ancestral polymorphism and adaptive evolution in the trichothecene mycotoxin gene cluster of phytopathogenic Fusarium. Proc. Natl. Acad. Sci. USA 99:9278-9283.

40. Xue, A. G., Armstrong, H. D., Voldeng, G., and Babcock, C. 2004. Comparative aggressiveness of isolates of Fusarium spp. causing head blight on wheat in Canada. Can. J. Plant Pathol. 26:81-88. 